\title{
Erratum to: FOXC1, a target of polycomb, inhibits metastasis of breast cancer cells
}

\author{
Juan $\mathrm{Du}^{1} \cdot \mathrm{Lin} \mathrm{Li}^{1} \cdot \mathrm{Zhouluo} \mathrm{Ou}^{2} \cdot$ Chenfei Kong ${ }^{3} \cdot \mathrm{Yu}_{\mathrm{Zhang}}{ }^{1} \cdot \mathrm{Zhixiong} \mathrm{Dong}^{1} \cdot$ \\ Shan $\mathrm{Zhu}^{3} \cdot$ Hao Jiang ${ }^{1} \cdot$ Zhimin Shao $^{2}$ - Baiqu Huang ${ }^{3}$ Jun Lu ${ }^{1}$
}

Published online: 8 April 2016

(C) Springer Science+Business Media New York 2016

\section{Erratum to: Breast Cancer Res Treat (2012) 131:65-73 DOI 10.1007/s10549-011-1396-3}

Unfortunately in the original publication of the article, the Fig. 2c (right panel), Fig. 5d and the caption of Fig. 5 were published erroneously. The corrected Figs. 2, 5 and caption of Fig. 5 are given in this erratum. The authors apologize for this error.
The online version of the original article can be found under doi:10.1007/s10549-011-1396-3.

Baiqu Huang

huangbq705@nenu.edu.cn

$\triangle$ Jun Lu

ycsuo@nenu.edu.cn

1 The Institute of Genetics and Cytology, Northeast Normal University, 5268 Renmin Street, Changchun 130024, China

2 Department of Oncology, Breast Cancer Institute, Cancer Hospital, Shanghai Medical College, Fudan University, Shanghai 200032, China

3 The Key Laboratory of Molecular Epigenetics of the Ministry of Education, Northeast Normal University, Changchun 130024, China

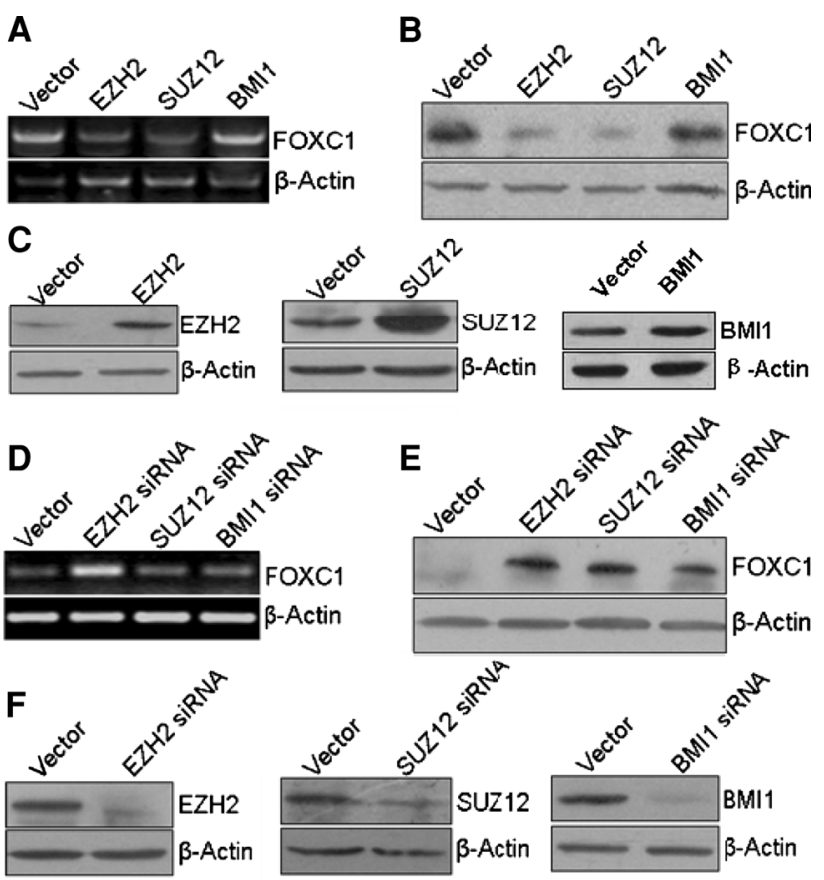

Fig. 2 Effects of overexpression and knockdown of PcG proteins on FOXC1 gene expression. MCF-7 cells were transfected with EZH2, SUZ12, and Bmi1 expression plasmids, and $48 \mathrm{~h}$ later the FOXC1 mRNA and protein levels were determined by PCR (a) and western blotting (b), respectively. The ectopic expression of EZH2, SUZ12, and Bmil proteins was confirmed by western blotting (c). MDA-MB231 cells were transfected with EZH2, SUZ12, and Bmi1 siRNA, and $48 \mathrm{~h}$ later RT-PCR and western blotting were performed. The endogenous FOXC1 mRNA (d) and protein (e) levels were upregulated. $\mathbf{f}$ Western blotting verification of the interfering efficiency of EZH2, SUZ12, and Bmi1 siRNAs in MDA-MB-231 cells 
A

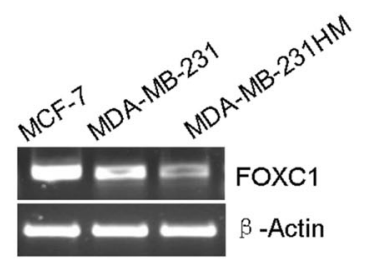

B

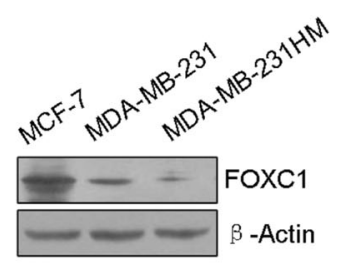

C MDA-MB-231HM

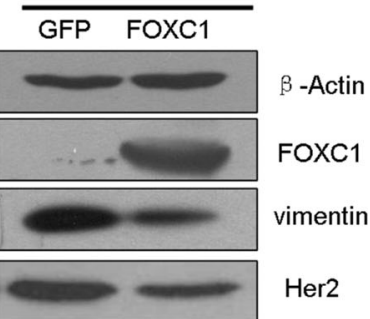

E

Serum-free L15 10\%FBSL15

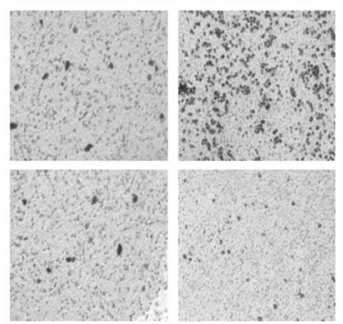

Fig. 5 FOXC1 prevented MDA-MB-231HM cell migration and invasion. FOXC1 expression was detected by RT-PCR (a) and western blotting (b) in MCF-7, MDA-MB-231 and MDA-MB231HM cell, respectively. $\mathbf{c}$ The whole cell lysates of FOXC1-MDAMB-231HM and GFP-MDA-MB-231HM were prepared for western
MDA-MB-231-GFP

MDA-MB-231-Foxc1

blotting detection of HER 2 and Vimentin. d FOXC1 prevented MDAMB-231HM cell migration. FOXC1-MDA-MB-231 and GFP-MDAMB-231 cells were plated in trans-well chambers as described above. e FOXC1 prevented MDA-MB-231HM cell invasion. Cell invasiveness was evaluated in vitro as described above 